\title{
ADAPTATION OF COURSE OF OPERATIONS RESEARCH TO NEEDS OF ENGINEERING STUDY PROGRAMMES BY INCLUDING SPECIFIC MODELS AND EXAMPLES
}

\author{
Svetlana Asmuss ${ }^{1}$, Natalja Budkina ${ }^{2}$ \\ ${ }^{1}$ University of Latvia, Latvia; ${ }^{2}$ Riga Technical University, Latvia \\ svetlana.asmuss@lu.lv, natalja.budkina@rtu.lv
}

\begin{abstract}
The course "Operations Research" or some parts of its themes are included in many study programmes. It is explained by a wide variety of applications of operations research models: operations research has been extensively used in engineering, industry, economics, management, business process, public administration, services and, of course, in agriculture, fisheries, mining, forestry, water resource system planning and management. Operations research in each of the mentioned application areas has its own history and traditions; special modifications of models and methodologies of operations research have been developed in the context of an application area. Successful teaching of the subject includes the real-world contexts, the usage in a particular specialty. The authors of this paper will share their long-term experience in teaching of "Operations Research", the main attention will be devoted to specific models and examples to be included in this course to adapt it to the needs of students of rural engineering specialities. Specific examples of production and service systems planning, networking, allocation and transportation problems, resource systems and supply chain planning and management, job scheduling, staff scheduling, queueing models and their illustrations could be considered as adaptation resources. The authors will discuss also how modelling tools such as JMT (Java Modelling Tools), QTP (Queueing ToolPak), CPN (Coloured Petri Nets) Tools, GeoGebra and Microsoft Project could be effectively used to support the study process.
\end{abstract}

Keywords: operations research, linear programming, networking, scheduling, queueing models.

\section{Introduction}

Operations research is a well-developed field with many techniques that are used to solve problems in a wide range of application areas. In its most basic form, operations research may be considered as an approach to solving problems in the management of complex systems, which includes the essential elements of the problem into a model and then analyses this model to obtain an optimal solution for implementation. Operations research deals with structuring, modelling and solving problems related to decision processes. To facilitate the choice and the implementation of more effective decisions operations research uses quantitative methods, models, techniques and algorithms from different scientific areas, where mathematics and informatics play a fundamental role, but we cannot discard statistics, economics, physics, engineering, sociology, psychology, etc.

Operations research has been extensively used in engineering, industry, economics, management, business process, public administration, services and, of course, in the natural resource sector, specifically in agriculture, fisheries, forestry, water resources system planning and management. The variety of areas of applications of operations research models and techniques explain, why the course "Operations Research" or some part of its themes is included in many study programmes. On the other hand, this variety should be taking into account when planning the course for each specific speciality due the limitation of the time for this course and the largest interest of the students to specifications of the course materials with respect to the fields of their speciality. The authors of this paper share their long-term experience in teaching of "Operations Research"; the main attention is devoted to specific models to be included in the course to adapt it to the needs of students of rural engineering specialities.

\section{Materials and methods}

The review of papers on operations research applications in the natural resource sector has been done with the aim to understand, which methods are most frequently used in the practice and what types of examples should be offered for students in the study course for rural engineering specialities. The references have been evaluated according to different operations research techniques with applications in such areas as agriculture, forestry, fisheries, water resources system planning and management. The observations show that almost all operations research methods have been applied for solving different decision making supporting problems in these areas. There are some common techniques in all of these areas, but at some level, these application fields are quite distinct: in the 
nature of the resources and the way they are handled, considered time horizons, planning and operating processes. We note that the mostly used methodologies for applications in the areas of rural engineering are the following: the classic optimization techniques, multiple criteria analysis, networking, scheduling and queueing models. In the next paragraphs, we discuss agriculture, forestry and fisheries separately; we mention important methods for each application area and consider some specific examples for students of the according speciality. We also give readers the references to the available papers with such type of examples. It should be noted that specific examples of production and service system planning, networking, allocation and transportation problems, resource systems and supply chain planning and management, job scheduling, staff scheduling, queueing models and their illustrations could be considered by using modelling and simulating tools such as Matlab Simulink, JMT (Java Modelling Tools), QTP (Queueing ToolPak), CPN (Coloured Petri Nets) Tools, GeoGebra and Microsoft Project. Some off these tools also are discussed in the paper.

\section{Agriculture}

Operations research models began to be applied in agriculture in the early 1950s. Waugh [14] is the first, who proposed the use of linear programming to establish least-cost combinations of feeding stuffs and livestock rations. The linear program minimizes the cost of the blend, while some specified level of nutritional requirements represents the model's constraints. From that time the linear programming is among the mostly used techniques of the operational research in agriculture.

Linear Programming is a constrained optimization technique, which optimizes some criterion within some constraints, where the objective function and constraints are linear:

$$
\begin{gathered}
F(x)=\sum_{j=1}^{n} c_{j} x_{j} \rightarrow \min \\
\left\{\begin{array}{l}
\sum_{j=1}^{n} a_{i j} x_{j} \geq b_{i}, \quad i=1, \ldots, m ; \\
x_{j} \geq 0, \quad j=1, \ldots, n .
\end{array}\right.
\end{gathered}
$$

The inequality type restrictions in (2) could be considered also as equalities for all or some $i$.

A typical example of the problem on mixtures, which could be suggested for students, is the following.

Example 1 (fertilizer mixtures). Suppose that there exist $n$ kinds of fertilizers, each of which contains $m$ elements of direct plant nutrition. Such elements can be nitrogen, phosphorus, potassium, magnesium, copper, manganese, etc. It is known that one unit of the $j$-th type of fertilizer $(j=1,2, \ldots$, $n$ ) contains $a_{i j}$ units of the $i$-th element $(i=1,2, \ldots, m)$ of the direct plant nutrition and has a cost $c_{j}$ It is necessary to make a mixed complex fertilizer, obtained by mechanical mixing of available fertilizers. In this case solving the problem (1)-(2) with respect to $x_{j}$, which denotes the amount of the $j$-th fertilizer used in the production of the fertilizer mixture, $(j=1,2, \ldots, n)$ guarantees the lowest cost of the fertilizer mixture with the following "chemical-economic" characteristic: the content of each $i$-th power element is not less than $b_{i}, i=1,2, \ldots, m$.

The next numerical example shows that compiling a diet can be considered in the context of the previous problem by including additional proportion constraints.

Example 2 (diet problem). A farm uses at least $400 \mathrm{~kg}$ of special feed daily. The special feed is a mixture of corn and soybean meal with the following compositions: $1 \mathrm{~kg}$ of corn consists of $0.09 \mathrm{~kg}$ protein and $0.02 \mathrm{~kg}$ fiber and costs $0.30 \mathrm{EUR} ; 1 \mathrm{~kg}$ of soybean meal consists of $0.6 \mathrm{~kg}$ protein and 0.06 $\mathrm{kg}$ fiber and costs $0.90 \mathrm{EUR}$. The dietary requirements of the special feed are at least $30 \%$ protein and at most $5 \%$ fiber. To determine the daily minimum-cost feed mix problem (1)-(2) can be applied with the objective function $F(x)=0.3 x_{1}+0.9 x_{2}$ and constraints $x_{1}+x_{2} \geq 400 ;-0.21 x_{1}+0.3 x_{2} \geq 0$; $0.03 x_{1}+0.01 x_{2} \geq 0$; (here the decision variables are $x_{1}$ and $x_{2}$ such that there are $x_{1} \mathrm{~kg}$ of corn and $x_{2} \mathrm{~kg}$ of soybean meal in the daily mix).

Specific interpretations of the profit maximization problem in agriculture and vegetable-growing industry can be considered. 
Example 3 (determining the structure of sown areas). In the vegetable-growing industry, the set of cultivated crops and the volumes of their production are determined by the availability of suitable land, the permissible labour costs, orders for certain types of crops, the demand for them, and the economic efficiency of production. When determining the structure of sown areas, it is necessary to ensure maximum economic efficiency based on available resources. Suppose that the following information is given: $n$ - the number of cultivated crops $(j=1, \ldots, n) ; m$ - the number of resources (land area, labor resources, etc.), $i=1, \ldots, m ; b_{i}$ - the volume of production resources of the $i$-th type; $a_{i j}$ - the cost of the $i$-th resource per 1 ha of the $j$-th culture crops; $c_{j}-$ the profit received from 1 ha of a crop of the $j$-th culture; $d_{j}$ - the volume of orders for $j$-th culture; $g_{j}$ - the maximum demand for the $j$ th culture; $u_{j}$-productivity of the $j$-th culture. The problem of maximization of the profit with respect to $x_{j}$, which denotes the area allocated for sowing the $j$-th crop, $j=1, \ldots, n$, can be formulated as

$$
\begin{gathered}
F(x)=\sum_{j=1}^{n} c_{j} x_{j} \rightarrow \max \\
\left\{\begin{array}{l}
\sum_{j=1}^{n} a_{i j} x_{j} \leq b_{i}, \quad i=1, \ldots, m ; \\
d_{j} \leq u_{j} x_{j} \leq g_{j}, \quad j=1, \ldots, n .
\end{array}\right.
\end{gathered}
$$

The next numerical example of the problem of optimal allocation of resources can be also written in the form (3)-(4).

Example 4 (optimal land and water resource allocation). A farmer has to plant two kinds of trees, say $\mathrm{A}$ and $\mathrm{B}$, on a land with $4400 \mathrm{sq} \mathrm{m}$ area. Each $\mathrm{A}$ tree requires at least $25 \mathrm{sq} \mathrm{m}$ of land, and B requires $40 \mathrm{sq} \mathrm{m}$. The annual water requirement of tree $\mathrm{A}$ is 30 units and that of $\mathrm{B}$ is 15 units, while at most 3300 units of water are available. It is estimated that the ratio of the number of B trees to the number of $A$ trees should not be less than $6 / 19$ and not be more that $17 / 8$. The return from one $\mathrm{B}$ tree is $50 \mathrm{EUR}$, while from one A tree it is one and a half times that of the return from B. Describe the plantation project of the farmer in terms of linear programming so that the return is maximum.

Detailed specific examples are considered in [13]: the first of them is applied for defining the optimal structure in the crop production by using the maximization gross margin as some set criteria, and the second is the problem of optimization a ration in the livestock production by minimization the least cost ration formulation as set criteria. In [12] the simplex algorithm solves the problem on the optimal land allocation of five food crops by using agriculture data, with respect to various factors, daily wages of labour and machine charges. We recommend also to take examples of the transportation problem by analogy with Example 9.

Taking into account a lot of limitations of linear programming sometimes the corresponding problems should be considered as problems of non-linear programming.

Example 5 (optimal sales plan). A farm sells the crop in $n$ ways: through the stores and through sales agents. When selling $x_{i}$ units through the $i$-th sales way, the expenses of sales are $f_{i}\left(x_{i}\right)$ EUR (for numerical illustration one can take $\left.f_{1}\left(x_{1}\right)=4 x_{1}+x_{1}{ }^{2}, f_{2}\left(x_{2}\right)=x_{2}{ }^{3}\right)$. Find the best way to sell the crop, minimizing total expenses of sales, if the total volume intended for sale is $a$ units. The mathematical model of this problem is as follows

$$
\begin{gathered}
F(x)=\sum_{i=1}^{n} f_{i}\left(x_{i}\right) \rightarrow \min \\
\left\{\begin{array}{l}
\sum_{i=1}^{n} x_{i}=a ; \\
x_{i} \geq 0, \quad i=1, \ldots, n .
\end{array}\right.
\end{gathered}
$$

The paper [5] presents a basic example illustrating the essentials of a non-linear mathematical programming model for a dairy farmer's insurance problem. The paper [8] considers the use of quadratic risk programming for whole-farm planning including a numerical example. 
It is self-evident nowadays that the effective functioning of an agricultural system involves considering biological, technical, private, social and environmental criteria, as well as resolving the conflicts inherent therein. Farmers do not seek to optimize a single objective function, but usually seek an optimal compromise between several conflicting objectives or try to establish satisfactory levels for their goals. Therefore, multiple criteria are frequently used in agricultural decision making. For the review of multi-criteria analysis applied to agricultural resource management the chapter "Dealing with Multiple Objectives in Agriculture" from [15] could be considered. The examples from Chapter 10 in [5] could be also used.

The problems of determination of optimum number of workers, flow of scheduled traffic, problems of loading and unloading, repairs and maintenance of equipment, etc. usually are considered by models of the queueing theory.

Example 6 (pure death model). A farm sells berries (flowers) in a shop. The shop stocks $a$ cwt of berries (pcs of flowers) at the beginning of a week. On the average, the shop sells $b$ cwt of berries (pcs of flowers) a day, but the actual demand follows a Poisson distribution. Whenever the stock level reaches $c$ cwt of berries (pcs of flowers), a new order of $a$ new cwt of berries (pcs of flowers) is placed for delivery at the beginning of the following week. Because of the nature of the item, all berries (flowers) left at the end of the week are disposed of. Determine the following: (a) the probability of placing an order in any day of the week; (b) the average number of cwt of berries (pcs of flowers) that will be discarded at the end of the week.

Example 7 (machine servicing model). An agricultural firm has $n$ harvesters and $m$ repairpersons. During the harvesting each machine is known to break down once every $a$ hours, on the average. It takes an average of $b$ hours to complete a repair. Both the time between breakdowns and the repair time follow the exponential distribution. Estimate the state of the park of harvesters in this situation. Determine the number of repairpersons needed to keep the harvesting running "smoothly".

We suggest applications of the game theory (especially, games with "nature") to be considered. In the context of games with "nature" the other player (playing against the farmer, who is to make decisions) can be the weather conditions, market conditions, insects, pests and diseases of plants and animals. One of the distinguishing features of models formed as games with "nature" is that it is only the farmer, who gains or loses; "nature" is passive in the sense of gains and losses to itself and it does not always try to do the worst to the farmer. The pay-off matrix in such model always is written from the viewpoint of the farmer and operations are done on the rows only (the rows correspond to strategies $S_{i}, i=1, \ldots, n$, of the farmer) and not on the columns (the columns correspond to states $T_{j}$, $j=1, \ldots, m$, of "nature").

Example 8 (game with "nature"). Suppose a farmer is faced with the decision as to whether he should take crop A or crop B. Further, assume that if it rains, taking crop A is the best proposition; but if he takes A and it does not rain, the crop fails entirely with no returns to him. The other crop B, does not give as good returns as A when it rains, but does give some returns even when there is "no rain".

Pay-off matrix example

Table 1

\begin{tabular}{|c|c|c|}
\cline { 2 - 3 } \multicolumn{1}{c|}{} & State of nature $T_{1}$ (rain) & State of nature $T_{2}$ (no rain) \\
\hline$S_{1}$ (take crop A) & 1 & 0 \\
\hline$S_{2}$ (take crop B) & $\alpha$ & $\beta$ \\
\hline
\end{tabular}

This example can be applied for demonstration of using different criteria of choice of the strategy: Wald's model (maxi-min criterion), Laplace's criterion (as application of the law of averages), Hurwicz (optimism-pessimism) criterion, minimum regret criterion of Savage (for more information and numerical examples of this approach, see, e.g., [1]).

The examples from another area of operations research - network scheduling - also could be presented in the context of agriculture. Network scheduling technique is used extensively to plan, schedule, and monitor large projects. The aim of it is to minimize trouble spots (such as delays, interruption, production bottlenecks, etc.) by identifying the critical factors. The different activities and their relationships of the entire project are represented diagrammatically with the help of networks 
and arrows, which is used for identifying critical activities and path. For corresponding examples see, e.g., [4].

\section{Forestry}

Operations research has been widely used in the forest management planning since the 1960s. The United States Forest Service implemented the first widely used linear program Timber RAM. Over the years, operational research techniques have been applied to support the forest products industry and public forestry organizations in their respective planning activities concerning the flow of wood fiber from the forest to the customer. The applications deal with a wide range of problems, ranging from long-term strategic problems related to forest management or company development to very shortterm operational problems, such as planning for real-time log/chip transportation or cutting. It should be noted that forest management planning decisions are often based on the forest owner's goals, which typically focus on economic criteria. Problems of forest management usually are considered as optimisation problems. Linear programming models have been the basic planning tools for native forests, which have multiple species coexisting in any area, standard tree rotations of 60 to 80 years, and planning horizons of several rotations. In the forestry sector, these include the following tasks: optimal design of forest machinery and equipment; rational use of raw materials and resources; nesting optimisation problems; optimization of the service system and maintenance of the machine and tractor fleet, etc. Transportation is usually an important cost in global logistics. Typically, logs are carried by trucks from forest locations to such destinations as pulp plants, sawmills, and ports for overseas transport.

Example 9 (optimisation of the timber transportation plan). A manager of a forest company must decide, how to supply the $m$ of their sawmills with wood, harvested in $n$ logging areas. The distances between the sawmills and logging areas are given as $c_{i j} \mathrm{~km}, i=1, \ldots, n, j=1, \ldots, m$. Transportation costs for wood transportation by timber trucks (one model) are proportional to the distance. Each plant requires continuous supply of wood and the minimum of daily supply of the $j$-th plant is $b_{j}, j=1, \ldots, m$, timber trucks. The daily maximum volume of cut wood for logging areas (in logging trucks) is given as $a_{i}, i=1, \ldots, n$. The mathematical model of this problem is

$$
\begin{gathered}
F(x)=\sum_{i=1}^{n} \sum_{j=1}^{m} c_{i j} x_{i j} \rightarrow \min \\
\left\{\begin{array}{l}
\sum_{j=1}^{m} x_{i j} \leq a_{i}, \quad i=1, \ldots n ; \\
x_{i j} \geq 0, \quad i=1, \ldots, n, j=1, \ldots, m ; \\
\sum_{i=1}^{n} x_{i j} \geq b_{j}, \quad j=1, \ldots, m ;
\end{array}\right.
\end{gathered}
$$

where integer variables $x_{i j} i=1, \ldots, n, j=1, \ldots, m$, describe the numbers of timber carriers shipped per day from the $i$-th logging areas to $j$-th sawmill.

Example 10 (rational use of raw materials and resources). A forestry has 24 ha of free land under steam and is interested in extracting income from it. It can grow conditional crop production, which reaches the commodity state for 1 year or bulls, taking a part of the land to pasture. Crop production is grown and sold in batches of $1000 \mathrm{~kg}$. It is required 1.5 ha for growing 1 batch of products and 4 ha for feeding one bull. The forestry can spend only 200 hours a year on its production. Suppose that it takes 20 hours to grow and harvest 1 batch of products. It takes 20 hours to care for one bull-calf. The forestry has the opportunity to spend 1000 EUR for this purpose. Annual costs for one batch of crop production are 3 EUR and 200 EUR per bull. Suppose that a contract has already been signed for the supply of two bull-calves. At current prices, $1 \mathrm{~kg}$ of products will bring a net income of $0.04 \mathrm{EUR}$, and one bull is 100 EUR. The problem can be reduced to maximization of the objective function $F(x)=100 x_{1}+0.04 x_{2}$ under constrains $x_{1}+x_{2} \leq 24 ; 200 x_{1}+3 x_{2} \leq 0 ; 20 x_{1}+20 x_{2} \leq 200 ; x_{1} \geq 2 ; x_{1} \geq 0$ where $x_{1}$ is the number of bull-calves (per year), $x_{2}$ is the number of crop production. The objective 
function shows annual net income, the restrictions are on the use of land, on the budget, on labour resources and the restriction under the contract.

We suggest to discuss in the context of forestry some problems by analogy with examples $3,4,5$, 7. Problems for determination of the number of trucks, queue at loading and unloading points could be considered as examples of linear programming tasks, queueing theory models or scheduling models.

Another important operation research application area is forestry supply chain design, planning and operation. The forestry supply chain involves all activities concerning the flow of fiber from the forest to the customer. D'Amours et al. [3] present an overview of problems arising in this supply chain, ranging from the long-term strategic rotations of forest growth, to the very short-term operational problems, as the lumber cutting stock problem, truck routing problems or finished product distribution.

One more area from forestry, where operations research techniques could be used, is the environmental question. In native forests in developed countries, sustainability, wildlife, biodiversity, and preservation of nature often play more important roles than timber production. In plantations, managers are increasingly concerned with protecting the soil and water quality. Spatial characterization of harvesting areas plays an important role in handling environmental concerns. In actual applications, analysts are using heuristic approaches, simulated annealing and Monte Carlo simulation. The linear programming and dynamic programming are used in more simple cases [9].

\section{Fisheries and aquaculture}

Many coastal areas are highly dependent on the fishing sector. This fact implies increasing efforts in collecting and collating more accurate data on the landings and discarding of fish, the state of fish stocks, and the economics of fishing fleets as a basis for improved bioeconomic analyses to support management decisions. Since 1950s operations research in fisheries has played a prominent role. Initial efforts were dedicated towards promoting stock conservation in the case of severely overexploited species. Researches from the 1970s extended the applications to estimations of technical efficiency of vessels, capacity utilization, fishermen's behavior and compliance. Operations research has explored diverse issues in fisheries management, both at a national and international level. In doing so, bioeconomic models (mathematical modeling that includes an economic model and the dynamics of living resources) have played a crucial role. The major operations research techniques for solving problems in fisheries are mathematical programming and optimisation based on statistical analysis, computer simulations, decision making based on multi criteria analysis. Similarly, operation research in aquaculture has combined modelling experiences from fisheries and other disciplines like agriculture and forestry to improve efficiency and economic gain at the farm and industry level. For application examples the review [2] could be used.

Taking into account that fish feed cost usually represents over $70 \%$ of the total cost of fish farm production and as a result an efficient feed practice is required for a sustainable fish industry, we suggest to include specific examples of linear programming for fish diet evaluation.

Example 11 (diet problem; see also Examples 1 and 2). The ingredients of a principal carp diet are groundnut cakes, soybean cakes, rice-bran, wheat bran, fish meal, brewer waste, til cakes; their market prices for $1 \mathrm{~kg}$ are given as $c_{j}$ EUR, $j=1, \ldots, 7$. The nutrients (protein, lipid, carbohydrate, calcium and phosphorous) contents of ingredients are known as $a_{i j} \%, i=1, \ldots, 5, j=1, \ldots, 7$. The minimum nutrient contents requirement for principal carp are given as $b_{j} \%, i=1, \ldots, 5$. Determine the composition of a principal carp diet using limited available feeds stuffs while satisfying nutrient and feed ingredient constraints, if the quantity of the mixture to be prepared is $d \mathrm{~kg}$. For more details, see [10].

Applications of the queueing theory in fisheries could be illustrated considering corresponding examples of the pure death model and the servicing model by analogy with Examples 6 and 7. The other types of queueing systems could be considered as applications, see, e.g., the next example on the design of fishing harbors.

Example 12 (fishing harbors planning). In order to determine an optimum number of berths of a fishing harbor the following statistical information was selected: the fishing vessels arrive at the harbor every $a$ hours on the average, and it takes an average of $b$ hours to upload each vessel by 
handling equipment. Both the interarrival and the service times are exponential with given parameters $\lambda$ and $\mu$. The restriction on waiting time is used for criteria of optimum.

\section{Modelling and simulating tools}

In this section we consider some tools, which could be used for solving and illustrating corresponding examples of applications.

There are many software packages available for solving linear programming problems (for example, Excel Solver, MathLab or Maple can be used in the study process). Taking into account that visualisation plays the essential role in understanding of the solution technique, we recommend to use GeoGebra - free software with interactive geometry, algebra, statistics and calculus applications intended for learning and teaching mathematics [6]. For geometrical illustration of a linear programming problem (in the case of two variables) by GeoGebra (see Fig. 1), it is necessary to constrain the feasible region and to find solutions by moving the straight line, which corresponds to the objective function level. The value of objective function changes on the screen of GeoGebra as the value of the slider according to the location of the objective function level line.

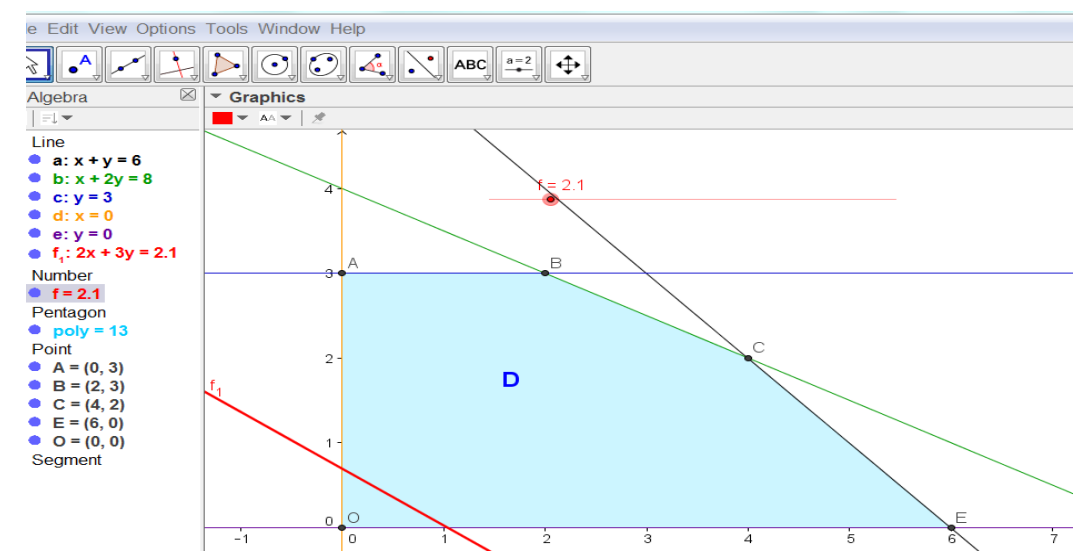

Fig. 1. Geometrical illustration of linear programming technique via GeoGebra

Special computer tools for queueing problems could be considered in the study course: Java Modelling Tools (JMT), Coloured Petri Net (CNP) tools, the Queueing ToolPak (QTP) and some others. We introduce them very shortly.

JMT [11] is a suite of applications developed by Politecnico di Milano and Imperial College London and released under GPL license. The project aims at offering a comprehensive framework for performance evaluation, system modeling with analytical and simulation techniques, capacity planning and workload characterization studies. The suite implements several state-of-the-art algorithms for the exact, approximate, asymptotic and simulative analysis of queueing network models. Models can be described either through wizard dialogs or with a graphical user-friendly interface. The application JSIMgraph is easier in the use for modelling a queueing system. After definition of all the necessary parameters, we could obtain the results of simulations as the mean values and as confidence intervals (see Fig. 2). The JMT gives also the possibility to conduct the analysis of changes of the performance measures in depending of the values of some other parameter of the system What-if-Analysis.

QTP is a Microsoft Excel add-in consisting of a library of functions that perform basic calculations for waiting line analysis. The functions allow integration of queueing performance measures into spreadsheet models without the limitations imposed by templates with fixed input and output areas that are commonly used for analysis of waiting lines. For example, the version 4.0 for waiting line systems consisting of a single waiting line, one or more parallel servers, a Poisson arrival process, exponentially distributed service durations, contains the functions for obtaining all primary performance measures: server utilization; average time spent in system (in queue) per customer; average number of customers in system (in queue).

The concept of CPN is an extended version of the classical Petri Net. CPN Tools is a discrete event modeling computer tool for CPN models supporting interactive and automatic simulations, supporting state spaces and performance analysis, combining CPN and the functional programming 
language CPN ML. By making simulations of a CPN model by using CPN Tools it is possible to investigate different scenarios and explore the behaviors of the system, to verify properties of the model by means of state space methods and model checking, and to conduct simulation-based performance analysis ([7]). Typical application domains of CPN models are the following: agent systems, business processes and workflows, data networks, communication protocols, embedded systems, manufacturing systems. CPN Tools could be used also for solution of such problems in agriculture, which require the technique of networks.

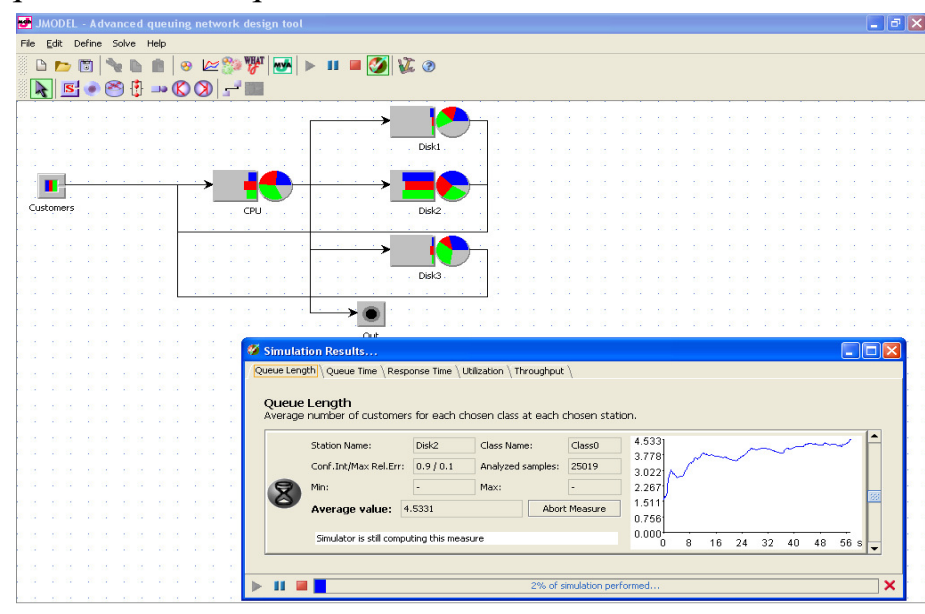

Fig. 2. Graphical representation of model in JSIMgraph and results of its simulations

We recommend also to represent scheduling and networking examples using the MS Project. MS Project is a project management software product developed and sold by Microsoft. The first commercial version was released for DOS in 1984. Now there are 20 million + users of this software package.

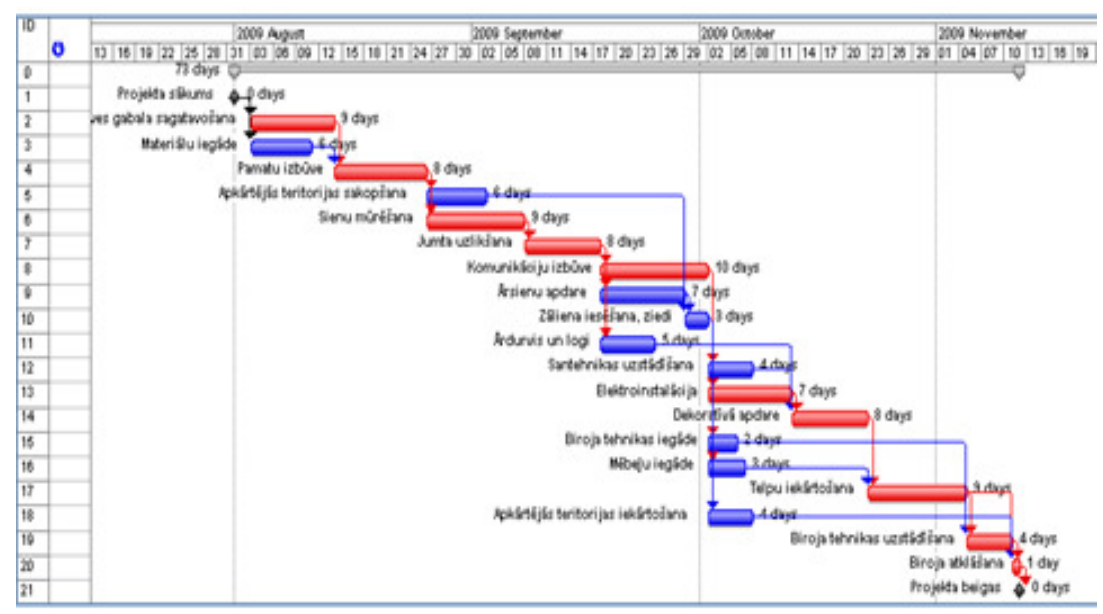

Fig. 3. Gantt chart in MS Project

It is designed to assist a project manager in developing a plan, assigning resources to tasks, tracking progress, managing the budget, analyzing workloads. The different activities and their relationships of the entire project are represented diagrammatically with the help of networks and arrows, used for identifying critical activities and path. Fig. 3 contains the Gantt chart - a display that shows activities on a graph, in which the time of each activity is represented.

\section{Conclusions}

Management of renewable natural resources in the areas of agriculture, fisheries and forestry is one of the fields of massive successful applications of different operations research models. The emergence of global, competitive markets has increased the need to derive efficient production processes, to reduce investment and operational costs, and to increase productivity. Consequently, better management practices will play an increasing vital role. On the other hand, the increasingly 
more complex environmental issues present additional challenges for operations research models. In this context, it is important to show for the students of the rural specialities already during their studies, which techniques of operations research and for which types of applications could be successfully used in their specialities.

\section{References}

[1] Agrawal R. C. Applications of operations research techniques in agriculture. Retrospective Theses and Dissertations. 3443, 1967. [10.03.2018]. Available at: http://lib.dr.iastate.edu/rtd/3443

[2] Bjørndal T., Lane D.E., Weintraub A. Operational research models and the management of fisheries and aquaculture: A review. European Journal of Operational Research, 156 (3), 2004, pp. 533-540.

[3] D'amours S., Rönnqvist M., Weintraub A. Using operational research for supply chain planningin the forest products industry. INFOR Information Systems and Operational Research, 2008, 46(4). pp. 265-281.

[4] Detlefsen N. K., Jensen A. L. Modelling optimal crop sequences using network flows. Agricultural Systems, 2007, 94(2): pp. 566-572, ISSN 0308-521X.

[5] Hardaker J. B., at el. Coping with risk in agriculture. 3rd Edition, Applied Decision Analysis, CABI Publishing, Printed and bound by Gutenberg Press Ltd, Tarxien, Malta, 2015.

[6] Introduction to GeoGebra. Version 4.4. [online] [10.03.2018]. Available at: http://static.geogebra.org/book/intro-en.pdf

[7] Jensen K., Kristensen L. Coloured Petri Nets. Modelling and Validation of Concurrent Systems. Springer-Verlag, 2009

[8] Kobzar O., Asseldonk M., Huirne R. Quadratic risk programming for whole-farm planning. Proceedings of 2nd International Conference for Young Researchers of Economics, 17-18 October 2002, Godollo, Hungary.

[9] Martell D., Gunn E., Weintraub A. Forest management challenges for operational researchers. European Journal of Operational Research, 104 (1), 1998, pp. 1-17.

[10] Nath T., Talukdar A. Linear programming technique in fish feed formulation. International Journal of Engineering Trends and Technology (IJETT) , v. 17, (3), 2014, pp. 132-135.

[11] Serazzi G. Ed. Performance evaluation modelling with JMT: learning by examples. Politecnico di Milano - DEI, TR 2008.09, 366 p.

[12] Sofi N. A., Ahmed A., Ahmad M., Bhat B. A. Decision making in agriculture: A linear programming approach. International Journal of Modern Mathematical Sciences, 2015, 13(2): p.p. 160-169.

[13] Vico G., Bodiroga R. Tools for planning in agriculture - Linear programming approach, AGRIBASE. Boosting Adult System Education in Agriculture, 2017.

[14] Waugh F. V. The minimum-cost dairy feed. Journal of Farm Economics 33, 1951, pp. 299-310.

[15] Weintraub A., Romero C., Bjørndal T., Epstein R., Miranda J. Handbook of operations research in natural resources. Springer, Boston, MA, 2007. 